\title{
LIE-TYPE DERIVATIONS OF FINITARY INCIDENCE ALGEBRAS
}

\author{
MYKOLA KHRYPCHENKO AND FENG WEI
}

\begin{abstract}
Let $P$ be an arbitrary partially ordered set, $R$ a commutative ring with identity and $F I(P, R)$ the finitary incidence algebra of $P$ over $R$. Under some natural assumption on $R$, we prove that each Lie-type derivation of $F I(P, R)$ is proper, which partially generalizes the main results of [39, 41].
\end{abstract}

\section{Introduction and Preliminaries}

Let $A$ be an associative algebra over a commutative ring $R$. We define the Lie product $[x, y]:=x y-y x$ and Jordan product $x \circ y:=x y+y x$ for all $x, y \in A$. Then $(A,[]$,$) becomes a Lie algebra and (A, \circ)$ is a Jordan algebra. It is a fascinating topic to study the connection between the associative, Lie and Jordan structures on $A$. In this field, two classes of mappings are of crucial importance. One of them consists of mappings, preserving a type of product, for example, Jordan homomorphisms and Lie homomorphisms. The other one is formed by differential operators, satisfying a type of Leibniz formulas, such as Jordan derivations and Lie derivations. In the AMS Hour Talk of 1961, Herstein proposed many problems concerning the structure of Jordan and Lie mappings in associative simple and prime rings [15]. Roughly speaking, he conjectured that these mappings are all of the proper or standard form. The renowned Herstein's Lie-type mapping research program was formulated since then. Martindale gave a major force in this program under the assumption that the rings contain some nontrivial idempotents [25]. The first idempotent-free result on Lie-type mappings was obtained by Brešar in [8]. We refer the reader to Brešar's survey paper [9] for a detailed historical background.

We recall that an $R$-linear mapping $d: A \rightarrow A$ is called a derivation if $d(x y)=$ $d(x) y+x d(y)$ for all $x, y \in A$, and it is called a Lie derivation if

$$
d([x, y])=[d(x), y]+[x, d(y)]
$$

for all $x, y \in A$. A Lie triple derivation is an $R$-linear mapping $d: A \rightarrow A$ which satisfies the rule

$$
d([[x, y], z])=[[d(x), y], z]+[[x, d(y)], z]+[[x, y], d(z)]
$$

for all $x, y, z \in A$. Obviously, a derivation is a Lie derivation, and similarly a Lie derivation is a Lie triple derivation. But, the converse statements are not true in general. For instance, suppose that $d: A \rightarrow A$ is a derivation and that $\tau: A \rightarrow Z(A)$ is a linear mapping from $A$ into its center $Z(A)$ such that $\tau([x, y])=0$ for all $x, y \in A$. Then $d+\tau$ is a Lie derivation of $A$, but it is not necessarily a derivation

Date: February 13, 2019.

2010 Mathematics Subject Classification. Primary 16W25; Secondary 16W10, 47L35.

Key words and phrases. Lie-type derivation, derivation, finitary incidence algebra. 
of $A$. Likewise, if $d: A \rightarrow A$ is a derivation and $\tau: A \rightarrow Z(A)$ is a linear mapping from $A$ into its center $Z(A)$ such that $\tau([[x, y], z])=0$ for all $x, y, z \in A$, then $d+\tau$ is a Lie triple derivation of $A$, but it is not necessarily a Lie derivation of $A$. In fact, if $d$ is a derivation of $A$ and $\tau$ is an $R$-linear mapping from $A$ into its center $Z(A)$, then $d+\tau$ is a Lie derivation (resp. Lie triple derivation) if and only if $\tau$ annihilates all commutators $[x, y]$ (resp. second commutators $[[x, y], z]$ ). A Lie derivation (resp. Lie triple derivation) of the form $d+\tau$, with $d$ being a derivation and $\tau$ a central-valued linear mapping annihilating each commutator (resp. second commutator), will be said to be proper.

Taking into account the definitions of Lie derivations and Lie triple derivations, one naturally expect to extend them in one more general way. Suppose that $n \geq 2$ is a fixed positive integer. Let us introduce a family of polynomials on $A$

$$
\begin{aligned}
p_{1}\left(x_{1}\right) & =x_{1} \\
p_{2}\left(x_{1}, x_{2}\right) & =\left[x_{1}, x_{2}\right] \\
p_{3}\left(x_{1}, x_{2}, x_{3}\right) & =\left[p_{2}\left(x_{1}, x_{2}\right), x_{3}\right]=\left[\left[x_{1}, x_{2}\right], x_{3}\right] \\
\vdots & \vdots \\
p_{n}\left(x_{1}, x_{2}, \cdots, x_{n}\right) & =\left[p_{n-1}\left(x_{1}, x_{2}, \cdots, x_{n-1}\right), x_{n}\right] .
\end{aligned}
$$

The polynomial $p_{n}\left(x_{1}, x_{2}, \cdots, x_{n}\right)$ is said to be an $(n-1)$-th commutator $(n \geq 2)$. An $R$-linear mapping $L: A \rightarrow A$ is called a Lie $n$-derivation if

$$
L\left(p_{n}\left(x_{1}, x_{2}, \ldots, x_{n}\right)\right)=\sum_{k=1}^{n} p_{n}\left(x_{1}, \ldots, x_{k-1}, L\left(x_{k}\right), x_{k+1}, \ldots, x_{n}\right)
$$

holds for all $x_{1}, x_{2}, \ldots, x_{n} \in A$. Lie $n$-derivations were introduced by Abdullaev [1]. In particular, he showed that every Lie $n$-derivation $L$ on a von Neumann algebra $M$ without central summands of type $I_{1}$ can be decomposed as $L=D+E$, where $D$ is an ordinary derivation on $M$ and $E$ is a center-valued mapping annihilating all $n$-th commutators. This result extends an older assertion (for $n=2,3$ ) due to Miers [27, 28]. By definition, a Lie derivation is a Lie 2-derivation and a Lie triple derivation is a Lie 3-derivation. It is straightforward to check that every Lie $n$-derivation on $A$ is a Lie $(n+k(n-1))$-derivation for all $k \in \mathbb{N}_{0}$. Lie 2derivations, Lie 3 -derivations and Lie $n$-derivations are collectively referred to as Lie-type derivations. We shall say that a Lie $n$-derivation of $A$ is proper, if $L=d+\tau$, where $d$ is a derivation of $A$ and $\tau$ is a central-valued linear mapping annihilating each $(n-1)$-th commutator $p_{n}\left(x_{1}, \ldots, x_{n}\right)$, where $x_{1}, \ldots, x_{n} \in A$. The question of whether each Lie-type derivation on a given algebra has the proper form is extensively studied, see [3-7, 12-14, 22-30, 36, 37, 40]. These works totally fulfill the Herstein's program in the background of triangular algebras and operator algebras. However, Cheung's work [12] is of special significance in our present case. He viewed the nest algebra over a Hilbert space as a triangular algebra and hence circumvented the analysis technique. This method makes it possible to study Lie derivations on incidence algebras in a combinatorial and linear manner [39, 41].

Let $(P, \leq)$ be a partially ordered set (poset) and $R$ a commutative ring with identity. With any pair of $x \leq y$ from $P$ associate a symbol $e_{x y}$ and denote by 
$I(P, R)$ the $R$-module of formal sums

$$
\alpha=\sum_{x \leq y} \alpha(x, y) e_{x y}
$$

where $\alpha(x, y) \in R$. If $x$ and $y$ run through a subset $X$ of the ordered pairs $x \leq y$ in the sum (2), then it is meant that $\alpha(x, y)=0$ for any pair $x \leq y$ which does not belong to $X$.

The sum (2) is called a finitary series [18], whenever for any pair of $x, y \in P$ with $x<y$ there exists only a finite number of $u, v \in P$, such that $x \leq u<v \leq y$ and $\alpha(u, v) \neq 0$. The set of finitary series, denoted by $F I(P, R)$, is an $R$-submodule of $I(P, R)$ which is closed under the convolution of the series:

$$
\alpha \beta=\sum_{x \leq y}\left(\sum_{x \leq z \leq y} \alpha(x, z) \beta(z, y)\right) e_{x y}
$$

for $\alpha, \beta \in F I(P, R)$. Thus, $F I(P, R)$ is an $R$-algebra, called the finitary incidence algebra of $P$ over $R$. Moreover, $I(P, R)$ is a bimodule over $F I(P, R)$ by means of of (3).

The incidence algebra of a poset was first considered by Ward in [38] as a generalized algebra of arithmetic functions. Rota and Stanley developed incidence algebras as fundamental structures of enumerative combinatorial theory and the allied areas of arithmetic function theory (see [34]). Furthermore, Stanley [33] initiated the study of algebraic mappings and combinatorial structure of an incidence algebra. Since then, the automorphisms and other algebraic mappings of incidence algebras have been increasingly significant (see [2, 10, 11, 16, 17, 19-21, 32, 39, 41] and the references therein). On the other hand, in the theory of operator algebras, the incidence algebra of a finite poset is referred as a bigraph algebra or a finite dimensional CSL algebra.

The main goal of this paper is to describe Lie-type derivations of the finitary incidence algebra $F I(P, R)$.

\section{LiE $n$-DERIVATIONS OF $F I(P, R)$}

We shall identify $e_{x y}$ with $1_{R} e_{x y}$. We shall also write $e_{x}$ for $e_{x x}$. Observe that $e_{x y} e_{u v}=\delta_{y u} e_{x v}$ by the definition of convolution. We shall also frequently use the formula

$$
e_{x} \alpha e_{y}=\alpha(x, y) e_{x y}
$$

for all $\alpha \in F I(P, R)$ and $x \leq y$.

Lemma 2.1. Let $\alpha \in F I(P, R)$. Then for all $x<y$

$$
\begin{aligned}
p_{n}\left(e_{x}, \alpha, e_{y}, \ldots, e_{y}\right) & =\alpha(x, y) e_{x y}, n \geq 3, \\
p_{n}\left(\alpha, e_{y}, \ldots, e_{y}\right)(x, y) & =\alpha(x, y), n \geq 2, \\
p_{n}\left(\alpha, e_{x y}, e_{y}, \ldots, e_{y}\right) & =(\alpha-\alpha(y, y)) e_{x y}, \quad n \geq 3 .
\end{aligned}
$$

Proof. For (5), we should remark that

$$
\left[e_{x} \beta, e_{y}\right]=e_{x} \beta e_{y}
$$


for any $\beta \in F I(P, R)$, which is due to the fact that $e_{y} e_{x}=0$. Since $e_{x} e_{y}=e_{y} \beta e_{x}=$ 0 , we further get $\left[\beta e_{x}, e_{y}\right]=0$. And hence

$$
p_{3}\left(e_{x}, \alpha, e_{y}\right)=\left[\left[e_{x}, \alpha\right], e_{y}\right]=\left[e_{x} \alpha, e_{y}\right]-\left[\alpha e_{x}, e_{y}\right]=e_{x} \alpha e_{y} .
$$

It follows from (8) that

$$
p_{4}\left(e_{x}, \alpha, e_{y}, e_{y}\right)=\left[p_{3}\left(e_{x}, \alpha, e_{y}\right), e_{y}\right]=\left[e_{x} \alpha e_{y}, e_{y}\right]=e_{x} \alpha e_{y} \cdot e_{y}=e_{x} \alpha e_{y} .
$$

By a trivial induction argument, the left-hand side of (5) coincides with $e_{x} \alpha e_{y}$. It remains to apply (4).

Now, to prove (6), let us write

$$
p_{2}\left(\alpha, e_{y}\right)(x, y)=\left[\alpha, e_{y}\right](x, y)=\left(\alpha e_{y}-e_{y} \alpha\right)(x, y)=\alpha(x, y),
$$

whence (6) by induction.

Finally, let us calculate (7) for $n=3$ :

$$
\begin{aligned}
p_{3}\left(\alpha, e_{x y}, e_{y}\right) & =\left(\alpha e_{x y}-e_{x y} \alpha\right) e_{y}-e_{y}\left(\alpha e_{x y}-e_{x y} \alpha\right) \\
& =\alpha e_{x y}-\alpha(y, y) e_{x y} \\
& =(\alpha-\alpha(y, y)) e_{x y} .
\end{aligned}
$$

Now since $(\alpha-\alpha(y, y)) e_{x y} \cdot e_{y}=(\alpha-\alpha(y, y)) e_{x y}$ and $e_{y} \cdot(\alpha-\alpha(y, y)) e_{x y}=0$, we obtain (7) by induction for all $n \geq 3$.

We shall need generalizations of Lemmas 2.3 and 3.4 from [41]. To this end, we shall slightly change the definition of the restriction used in [41], as we did in [20].

Definition 2.2. For any $\alpha \in F I(P, R)$ and $x \leq y$, we define the restriction of $\alpha$ to $[x, y]=\{z \in X \mid x \leq z \leq y\}$ to be

$$
\left.\alpha\right|_{x} ^{y}=\alpha(x, y) e_{x y}+\sum_{x \leq v<y} \alpha(x, v) e_{x v}+\sum_{x<u \leq y} \alpha(u, y) e_{u y} .
$$

Clearly, the mapping $\left.\alpha \mapsto \alpha\right|_{x} ^{y}$ is linear. Moreover, we have the next lemma, whose proof is straightforward.

Lemma 2.3. For any $\alpha \in F I(P, R)$, we have

(i) $\left.\left(\left.\alpha\right|_{x} ^{y}\right)\right|_{x} ^{y}=\left.\alpha\right|_{x} ^{y}$;

(ii) $(\alpha \beta)(x, y)=\left(\left.\alpha\right|_{x} ^{y} \beta\right)(x, y)=\left(\left.\alpha \beta\right|_{x} ^{y}\right)(x, y)$.

Lemma 2.4. Let $L$ be a Lie $n$-derivation of $F I(P, R)$, where $n \geq 2$, and $x<y$. Then

$$
L(\alpha)(x, y)=L\left(\left.\alpha\right|_{x} ^{y}\right)(x, y) .
$$

Proof. It suffices to verify the case $n \geq 3$, since each Lie derivation is a Lie triple derivation. We shall use (1), (5) and (6):

$$
\begin{aligned}
L(\alpha)(x, y)= & p_{n}\left(e_{x}, L(\alpha), e_{y}, \ldots, e_{y}\right)(x, y) \\
= & L\left(p_{n}\left(e_{x}, \alpha, e_{y}, \ldots, e_{y}\right)\right)(x, y)-p_{n}\left(L\left(e_{x}\right), \alpha, e_{y}, \ldots, e_{y}\right)(x, y) \\
& -p_{n}\left(e_{x}, \alpha, L\left(e_{y}\right), e_{y}, \ldots e_{y}\right)(x, y) \\
& -\sum_{i=1}^{n-3} p_{n}(e_{x}, \alpha, \underbrace{e_{y}, \ldots, e_{y}}_{i}, L\left(e_{y}\right), e_{y}, \ldots, e_{y})(x, y) \\
= & \alpha(x, y) L\left(e_{x y}\right)(x, y)-p_{2}\left(L\left(e_{x}\right), \alpha\right)(x, y)
\end{aligned}
$$




$$
-p_{3}\left(e_{x}, \alpha, L\left(e_{y}\right)\right)(x, y)-(n-3) p_{2}\left(\alpha(x, y) e_{x y}, L\left(e_{y}\right)\right)(x, y) .
$$

Taking into account the fact that $\alpha(x, y)=\left.\alpha\right|_{x} ^{y}(x, y)$ together with Lemma 2.3 (ii), we arrive at

$$
\begin{aligned}
p_{2}\left(L\left(e_{x}\right), \alpha\right)(x, y) & =\left(L\left(e_{x}\right) \alpha-\alpha L\left(e_{x}\right)\right)(x, y) \\
& =\left(\left.L\left(e_{x}\right) \alpha\right|_{x} ^{y}-\left.\alpha\right|_{x} ^{y} L\left(e_{x}\right)\right)(x, y) \\
& =p_{2}\left(L\left(e_{x}\right),\left.\alpha\right|_{x} ^{y}\right)(x, y) .
\end{aligned}
$$

Furthermore, using Lemma 2.3 (ii) once again, we have

$$
\begin{aligned}
p_{3}\left(e_{x}, \alpha, L\left(e_{y}\right)\right)(x, y) & =\left(\left(e_{x} \alpha-\alpha e_{x}\right) L\left(e_{y}\right)-L\left(e_{y}\right)\left(e_{x} \alpha-\alpha e_{x}\right)\right)(x, y) \\
& =\left(\alpha L\left(e_{y}\right)\right)(x, y)-\alpha(x, x) L\left(e_{y}\right)(x, y)-L\left(e_{y}\right)(x, x) \alpha(x, y) \\
& =\left(\left.\alpha\right|_{x} ^{y} L\left(e_{y}\right)\right)(x, y)-\left.\alpha\right|_{x} ^{y}(x, x) L\left(e_{y}\right)(x, y)-\left.L\left(e_{y}\right)(x, x) \alpha\right|_{x} ^{y}(x, y) \\
& =p_{3}\left(e_{x},\left.\alpha\right|_{x} ^{y}, L\left(e_{y}\right)\right)(x, y) .
\end{aligned}
$$

Thus, we see that one can replace $\alpha$ by $\left.\alpha\right|_{x} ^{y}$ in the right-hand side of (11), whence (10).

Lemma 2.5. Let $L$ be a Lie $n$-derivation of $F I(P, R)$, where $n \geq 2$, and $u<x<$ $y<v$. Then

$$
\begin{aligned}
& L\left(e_{x y}\right)(x, v)=L\left(e_{y}\right)(y, v), \\
& L\left(e_{x y}\right)(u, y)=L\left(e_{x}\right)(u, x) .
\end{aligned}
$$

Proof. For (12), apply $L$ to the equality

$$
e_{x y}=p_{n}\left(e_{x y}, e_{y}, \ldots, e_{y}\right)
$$

and use (1) to get

$$
\begin{aligned}
L\left(e_{x y}\right)= & L\left(p_{n}\left(e_{x y}, e_{y}, \ldots, e_{y}\right)\right) \\
= & p_{n}\left(L\left(e_{x y}\right), e_{y}, \ldots, e_{y}\right)+p_{n}\left(e_{x y}, L\left(e_{y}\right), e_{y}, \ldots, e_{y}\right) \\
& +\sum_{i=0}^{n-3} p_{n}(e_{x y}, e_{y}, \ldots, e_{y}, L\left(e_{y}\right), \underbrace{e_{y}, \ldots, e_{y}}_{i}) \\
= & p_{n}\left(L\left(e_{x y}\right), e_{y}, \ldots, e_{y}\right)+\sum_{i=0}^{n-2} p_{i+2}(e_{x y}, L\left(e_{y}\right), \underbrace{e_{y}, \ldots, e_{y}}_{i}) .
\end{aligned}
$$

Observe that $\left[\beta, e_{y}\right](x, v)=0$ for an arbitrary $\beta \in F I(P, R)$, since $y \notin\{x, v\}$. Hence, taking the values of the both sides of $(15)$ at $(x, v)$, we obtain

$$
L\left(e_{x y}\right)(x, v)=p_{2}\left(e_{x y}, L\left(e_{y}\right)\right)(x, v)=\left(e_{x y} L\left(e_{y}\right)-L\left(e_{y}\right) e_{x y}\right)(x, v)=L\left(e_{y}\right)(y, v),
$$

proving (12).

By invoking $e_{x y}=(-1)^{n+1} p_{n}\left(e_{x y}, e_{x}, \ldots, e_{x}\right)$, one can show (13) in the same way as (12).

Lemma 2.6. Let $L$ be a Lie $n$-derivation of $F I(P, R)$ and $\alpha \in F I(P, R)$. If $R$ is $(n-1)$-torsion free, then

$$
L(\alpha)(x, x)=L(\alpha)(y, y)
$$

for all $x<y$. 
Proof. Applying $L$ to $p_{n}\left(\alpha, e_{x y}, e_{y}, \ldots, e_{y}\right)$ and using (1), we have

$$
\begin{aligned}
L\left(p_{n}\left(\alpha, e_{x y}, e_{y}, \ldots, e_{y}\right)\right)= & p_{n}\left(L(\alpha), e_{x y}, e_{y}, \ldots, e_{y}\right)+p_{n}\left(\alpha, L\left(e_{x y}\right), e_{y}, \ldots, e_{y}\right) \\
& +p_{n}\left(\alpha, e_{x y}, L\left(e_{y}\right), e_{y}, \ldots, e_{y}\right) \\
& +\sum_{i=1}^{n-3} p_{n}(\alpha, e_{x y}, \underbrace{e_{y}, \ldots, e_{y}}_{i}, L\left(e_{y}\right), e_{y}, \ldots, e_{y})
\end{aligned}
$$

where the summands (17) and (18) do not appear for $n=2$.

By (6) and (14), we get

$$
\begin{aligned}
p_{n}\left(L(\alpha), e_{x y}, e_{y}, \ldots, e_{y}\right)(x, y) & =p_{2}\left(L(\alpha), e_{x y}\right)(x, y) \\
& =\left(L(\alpha) e_{x y}-e_{x y} L(\alpha)\right)(x, y) \\
& =L(\alpha)(x, x)-L(\alpha)(y, y)
\end{aligned}
$$

Furthermore, using (6), we have

$$
p_{n}\left(\alpha, L\left(e_{x y}\right), e_{y}, \ldots, e_{y}\right)(x, y)=p_{2}\left(\alpha, L\left(e_{x y}\right)\right)(x, y)=\left(\alpha L\left(e_{x y}\right)-L\left(e_{x y}\right) \alpha\right)(x, y) .
$$

In view of (10), we know that

$$
\begin{aligned}
\left(\alpha L\left(e_{x y}\right)\right)(x, y) & =\sum_{x \leq z \leq y} \alpha(x, z) L\left(e_{x y}\right)(z, y) \\
& =\sum_{x \leq z \leq y} \alpha(x, z) L\left(\left.e_{x y}\right|_{z} ^{y}\right)(z, y) \\
& =\alpha(x, x) L\left(e_{x y}\right)(x, y),
\end{aligned}
$$

which is due to $\left.e_{x y}\right|_{z} ^{y}=\delta_{x z} e_{x y}$ for $x \leq z \leq y$. In an analogous manner, one can show

$$
\left(L\left(e_{x y}\right) \alpha\right)(x, y)=\alpha(y, y) L\left(e_{x y}\right)(x, y) .
$$

Considering (6), we have

$$
\begin{aligned}
p_{n}\left(\alpha, e_{x y}, L\left(e_{y}\right), e_{y}, \ldots, e_{y}\right)(x, y)= & p_{3}\left(\alpha, e_{x y}, L\left(e_{y}\right)\right)(x, y) \\
= & \left(\left(\alpha e_{x y}-e_{x y} \alpha\right) L\left(e_{y}\right)-L\left(e_{y}\right)\left(\alpha e_{x y}-e_{x y} \alpha\right)\right)(x, y) \\
= & \alpha(x, x) L\left(e_{y}\right)(y, y)-\alpha(y, y) L\left(e_{y}\right)(y, y) \\
& -L\left(e_{y}\right)(x, x) \alpha(x, x)+L\left(e_{y}\right)(x, x) \alpha(y, y) \\
= & (\alpha(x, x)-\alpha(y, y))\left(L\left(e_{y}\right)(y, y)-L\left(e_{y}\right)(x, x)\right) .
\end{aligned}
$$

Finally, by invoking (6) and (7) we assert that

$$
\begin{aligned}
p_{n}(\alpha, e_{x y}, \underbrace{e_{y}, \ldots, e_{y}}_{i}, L\left(e_{y}\right), e_{y}, \ldots, e_{y})(x, y) & =p_{i+3}(\alpha, e_{x y}, \underbrace{e_{y}, \ldots, e_{y}}_{i}, L\left(e_{y}\right))(x, y) \\
& =p_{2}\left((\alpha-\alpha(y, y)) e_{x y}, L\left(e_{y}\right)\right)(x, y),
\end{aligned}
$$

the latter being

$$
\begin{aligned}
& \left((\alpha-\alpha(y, y)) e_{x y} L\left(e_{y}\right)-L\left(e_{y}\right)(\alpha-\alpha(y, y)) e_{x y}\right)(x, y) \\
& \quad=(\alpha(x, x)-\alpha(y, y)) L\left(e_{y}\right)(y, y)-L\left(e_{y}\right)(x, x)(\alpha(x, x)-\alpha(y, y)) \\
& \quad=(\alpha(x, x)-\alpha(y, y))\left(L\left(e_{y}\right)(y, y)-L\left(e_{y}\right)(x, x)\right) .
\end{aligned}
$$

On the other hand, by (7), (9) and (10), we arrive at

$$
L\left(p_{n}\left(\alpha, e_{x y}, e_{y}, \ldots, e_{y}\right)\right)(x, y)=L\left((\alpha-\alpha(y, y)) e_{x y}\right)(x, y)
$$




$$
\begin{aligned}
& =L\left(\left.\left((\alpha-\alpha(y, y)) e_{x y}\right)\right|_{x} ^{y}\right)(x, y) \\
& =L\left(\alpha(x, x) e_{x y}-\alpha(y, y) e_{x y}\right)(x, y) \\
& =(\alpha(x, x)-\alpha(y, y)) L\left(e_{x y}\right)(x, y) .
\end{aligned}
$$

Thus, taking the values of the both sides of $(18)$ at $(x, y)$, we obtain

$$
\begin{aligned}
(\alpha(x, x)-\alpha(y, y)) L\left(e_{x y}\right)(x, y)= & L(\alpha)(x, x)-L(\alpha)(y, y) \\
& +(\alpha(x, x)-\alpha(y, y)) L\left(e_{x y}\right)(x, y) \\
& +(n-2)(\alpha(x, x)-\alpha(y, y))\left(L\left(e_{y}\right)(y, y)-L\left(e_{y}\right)(x, x)\right),
\end{aligned}
$$

whence

$$
L(\alpha)(x, x)-L(\alpha)(y, y)=(n-2)(\alpha(x, x)-\alpha(y, y))\left(L\left(e_{y}\right)(x, x)-L\left(e_{y}\right)(y, y)\right) .
$$

If $n=2$, then we immediately obtain (16). If $n \geq 3$, then taking $\alpha=e_{y}$ in (19), we get

$$
L\left(e_{y}\right)(x, x)-L\left(e_{y}\right)(y, y)=-(n-2)\left(L\left(e_{y}\right)(x, x)-L\left(e_{y}\right)(y, y)\right),
$$

i.e.

$$
(n-1)\left(L\left(e_{y}\right)(x, x)-L\left(e_{y}\right)(y, y)\right)=0 .
$$

If $R$ is $(n-1)$-torsion free, the latter yields $L\left(e_{y}\right)(x, x)-L\left(e_{y}\right)(y, y)=0$, proving thus (16).

Definition 2.7. Given $\alpha \in F I(P, R)$, define the diagonal of $\alpha$ to be

$$
\alpha_{D}=\sum_{x \in P} \alpha(x, x) e_{x x} \in F I(P, R) .
$$

An element $\alpha \in F I(P, R)$ is said to be diagonal whenever $\alpha=\alpha_{D}$.

Recall from [31] that the center $Z(F I(P, R))$ of $F I(P, R)$ consists of diagonal elements $\alpha \in F I(P, R)$, such that $\alpha(x, x)=\alpha(y, y)$ for all $x<y$ in $P$.

Corollary 2.8. Let $L$ be a Lie $n$-derivation of $F I(P, R)$ and $\alpha \in F I(P, R)$. If $R$ is $(n-1)$-torsion free, then $L(\alpha)_{D} \in Z(F I(P, R))$.

Lemma 2.9. Let $L$ be a Lie $n$-derivation of $F I(P, R)$. If $R$ is $(n-1)$-torsion free, then for all $x<y<z$ :

$$
\begin{aligned}
L\left(e_{x}\right)(x, y)+L\left(e_{y}\right)(x, y) & =0, \\
L\left(e_{x y}\right)(x, y)+L\left(e_{y z}\right)(y, z) & =L\left(e_{x z}\right)(x, z) .
\end{aligned}
$$

Proof. Since $e_{x} e_{y}=e_{y} e_{x}=0$, we see that $p_{n}\left(e_{x}, e_{y}, \ldots, e_{y}\right)=0$. We therefore have by (1)

$$
\begin{aligned}
0= & p_{n}\left(L\left(e_{x}\right), e_{y}, \ldots, e_{y}\right)+p_{n}\left(e_{x}, L\left(e_{y}\right), e_{y} \ldots, e_{y}\right) \\
& +\sum_{i=1}^{n-2} p_{n}(e_{x}, \underbrace{e_{y} \ldots, e_{y}}_{i}, L\left(e_{y}\right), e_{y} \ldots, e_{y}) \\
= & p_{n}\left(L\left(e_{x}\right), e_{y}, \ldots, e_{y}\right)+p_{n}\left(e_{x}, L\left(e_{y}\right), e_{y} \ldots, e_{y}\right) .
\end{aligned}
$$

By (5) and (6) the value of (22) at $(x, y)$ equals $L\left(e_{x}\right)(x, y)+L\left(e_{y}\right)(x, y)$, whence (20).

To prove (21), observe that

$$
e_{x z}=p_{n-1}\left(e_{x z}, e_{z} \ldots, e_{z}\right)=p_{n}\left(e_{x y}, e_{y z}, e_{z} \ldots, e_{z}\right) .
$$


Thus

$$
\begin{aligned}
L\left(e_{x z}\right)= & L\left(p_{n}\left(e_{x y}, e_{y z}, e_{z} \ldots, e_{z}\right)\right) \\
= & p_{n}\left(L\left(e_{x y}\right), e_{y z}, e_{z} \ldots, e_{z}\right)+p_{n}\left(e_{x y}, L\left(e_{y z}\right), e_{z} \ldots, e_{z}\right) \\
& +\sum_{i=0}^{n-3} p_{n}(e_{x y}, e_{y z}, \underbrace{e_{z} \ldots, e_{z}}_{i}, L\left(e_{z}\right), e_{z} \ldots, e_{z}) .
\end{aligned}
$$

It follows from (6) that

$$
\begin{aligned}
p_{n}\left(L\left(e_{x y}\right), e_{y z}, e_{z} \ldots, e_{z}\right)(x, z) & =p_{2}\left(L\left(e_{x y}\right), e_{y z}\right)(x, z) \\
& =\left(L\left(e_{x y}\right) e_{y z}-e_{y z} L\left(e_{x y}\right)\right)(x, z) \\
& =L\left(e_{x y}\right)(x, y) .
\end{aligned}
$$

Similarly, we get

$$
\begin{aligned}
p_{n}\left(e_{x y}, L\left(e_{y z}\right), e_{z} \ldots, e_{z}\right)(x, z) & =p_{2}\left(e_{x y}, L\left(e_{y z}\right)\right)(x, z) \\
& =\left(e_{x y} L\left(e_{y z}\right)-L\left(e_{y z}\right) e_{x y}\right)(x, z) \\
& =L\left(e_{y z}\right)(y, z) .
\end{aligned}
$$

Now, using (6), (14) and (23), we obtain

$$
\begin{aligned}
p_{n}(e_{x y}, e_{y z}, \underbrace{e_{z} \ldots, e_{z}}_{i}, L\left(e_{z}\right), e_{z} \ldots, e_{z})(x, z) & =p_{2}\left(e_{x z}, L\left(e_{z}\right)\right)(x, z) \\
& =\left(e_{x z} L\left(e_{z}\right)-L\left(e_{z}\right) e_{x z}\right)(x, z) \\
& =L\left(e_{z}\right)(x, x)-L\left(e_{z}\right)(z, z) .
\end{aligned}
$$

The latter is zero by Lemma 2.6. Thus, (21) is proved by taking the values of the both sides of $(24)$ at $(x, z)$.

Definition 2.10. Let $L$ be a Lie $n$-derivation of $F I(P, R)$ and $\alpha \in F I(P, R)$. Define

$$
\begin{aligned}
\tau(\alpha) & =L(\alpha)_{D}, \\
d(\alpha) & =L(\alpha)-L(\alpha)_{D} .
\end{aligned}
$$

Lemma 2.11. Let $L$ be a Lie n-derivation of $F I(P, R)$ and $\tau$ be given by (25). If $R$ is $(n-1)$-torsion free, then $\tau$ is a central-valued linear mapping which annihilates all the $n$-th commutators in $F I(P, R)$.

Proof. We have already seen in Corollary 2.8 that $L(\alpha)_{D} \in Z(F I(P, R))$, so $\tau$ is center-valued. Since $L$ is linear, then $\tau$ is also linear. Moreover, for any $\alpha_{1}, \ldots, \alpha_{n} \in$ $F I(P, R)$, one has by (1) and (25) and the easy fact that $(\alpha \beta)_{D}=\alpha_{D} \beta_{D}$ :

$$
\begin{aligned}
\tau\left(p_{n}\left(\alpha_{1}, \ldots, \alpha_{n}\right)\right) & =L\left(p_{n}\left(\alpha_{1}, \ldots, \alpha_{n}\right)\right)_{D} \\
& =\left(\sum_{i=1}^{n} p_{n}\left(\alpha_{1}, \ldots, L\left(\alpha_{i}\right), \ldots, \alpha_{n}\right)\right)_{D} \\
& =\sum_{i=1}^{n} p_{n}\left(\left(\alpha_{1}\right)_{D}, \ldots, L\left(\alpha_{i}\right)_{D}, \ldots,\left(\alpha_{n}\right)_{D}\right)
\end{aligned}
$$

which is zero, as diagonal elements commute.

Lemma 2.12. If $R$ is $(n-1)$-torsion free, then $d$ is a derivation of $F I(P, R)$. 
Proof. We need to prove that

$$
d(\alpha \beta)=d(\alpha) \beta+\alpha d(\beta)
$$

for all $\alpha, \beta \in F I(P, R)$. Observe that

$$
d(\alpha)_{D}=L(\alpha)_{D}-L(\alpha)_{D}=0 .
$$

So (27) trivially holds at $(x, x)$ for all $x \in P$. Now, given $x<y$, by (10), (25), (26) and (28) we have

$$
\begin{aligned}
(d(\alpha) \beta)(x, y)= & \sum_{x \leq z \leq y} d(\alpha)(x, z) \beta(z, y) \\
= & \sum_{x<z \leq y} L(\alpha)(x, z) \beta(z, y)=\sum_{x<z \leq y} L\left(\left.\alpha\right|_{x} ^{z}\right)(x, z) \beta(z, y) \\
= & \sum_{x<z \leq y}\left(\alpha(x, z) L\left(e_{x z}\right)(x, z)+\sum_{x \leq v<z} \alpha(x, v) L\left(e_{x v}\right)(x, z)\right. \\
& \left.+\sum_{x<u \leq z} \alpha(u, z) L\left(e_{u z}\right)(x, z)\right) \beta(z, y) .
\end{aligned}
$$

Similarly,

$$
\begin{aligned}
(\alpha d(\beta))(x, y)= & \sum_{x \leq z \leq y} \alpha(x, z) d(\beta)(z, y) \\
= & \sum_{x \leq z<y} \alpha(x, z) L(\beta)(z, y)=\sum_{x \leq z<y} \alpha(x, z) L\left(\left.\beta\right|_{z} ^{y}\right)(z, y) \\
= & \sum_{x \leq z<y} \alpha(x, z)\left(\beta(z, y) L\left(e_{z y}\right)(z, y)+\sum_{z \leq v<y} \beta(z, v) L\left(e_{z v}\right)(z, y)\right. \\
& \left.+\sum_{z<u \leq y} \beta(u, y) L\left(e_{u y}\right)(z, y)\right) .
\end{aligned}
$$

Hence, adding (29) and (30), we arrive at

$$
\begin{aligned}
(d(\alpha) \beta+\alpha d(\beta))(x, y)= & (\alpha(x, y) \beta(y, y)+\alpha(x, x) \beta(x, y)) L\left(e_{x y}\right)(x, y) \\
& +\sum_{x<z<y} \alpha(x, z) \beta(z, y)\left(L\left(e_{x z}\right)(x, z)+L\left(e_{z y}\right)(z, y)\right) \\
& +\sum_{x<u \leq z \leq y} \alpha(u, z) \beta(z, y) L\left(e_{u z}\right)(x, z) \\
& +\sum_{x \leq z \leq v<y} \alpha(x, z) \beta(z, v) L\left(e_{z v}\right)(z, y) \\
& +\sum_{x \leq v<z \leq y} \alpha(x, v) \beta(z, y) L\left(e_{x v}\right)(x, z) \\
& +\sum_{x \leq z<u \leq y} \alpha(x, z) \beta(u, y) L\left(e_{u y}\right)(z, y) .
\end{aligned}
$$

The sum of (31) and (32) equals

$$
\begin{aligned}
& \left(\alpha(x, y) \beta(y, y)+\alpha(x, x) \beta(x, y)+\sum_{x<z<y} \alpha(x, z) \beta(z, y)\right) L\left(e_{x y}\right)(x, y) \\
& =(\alpha \beta)(x, y) L\left(e_{x y}\right)(x, y),
\end{aligned}
$$


which is due to (21).

Now,

$$
L\left(e_{u z}\right)(x, z)=L\left(e_{u}\right)(x, u)=L\left(e_{u y}\right)(x, y)
$$

for all $x<u<z \leq y$ and

$$
L\left(e_{z y}\right)(x, y)=L\left(e_{z}\right)(x, z)
$$

for all $x<z<y$ by (13). So (33) becomes

$$
\begin{aligned}
& \alpha(y, y) \beta(y, y) L\left(e_{y}\right)(x, y)+\sum_{x<z<y} \alpha(z, z) \beta(z, y) L\left(e_{z}\right)(x, z) \\
& \quad+\sum_{x<u<z \leq y} \alpha(u, z) \beta(z, y) L\left(e_{u z}\right)(x, z) \\
& =\alpha(y, y) \beta(y, y) L\left(e_{y}\right)(x, y)+\sum_{x<z<y} \alpha(z, z) \beta(z, y) L\left(e_{z y}\right)(x, y) \\
& \quad+\sum_{x<u<z \leq y} \alpha(u, z) \beta(z, y) L\left(e_{u y}\right)(x, y) \\
& =\sum_{x<u \leq z \leq y} \alpha(u, z) \beta(z, y) L\left(e_{u y}\right)(x, y)=\sum_{x<u \leq y}(\alpha \beta)(u, y) L\left(e_{u y}\right)(x, y)
\end{aligned}
$$

Similarly, (34) is equal to

$$
\sum_{x \leq v<y}(\alpha \beta)(x, v) L\left(e_{x v}\right)(x, y) .
$$

Adding (35) and (36) and making changes of variables in the both sums, we obtain

$$
\sum_{x \leq a<b \leq y} \alpha(x, a) \beta(b, y)\left(L\left(e_{x a}\right)(x, b)+L\left(e_{b y}\right)(a, y)\right)
$$

But

$$
L\left(e_{x a}\right)(x, b)+L\left(e_{b y}\right)(a, y)=L\left(e_{a}\right)(a, b)+L\left(e_{b}\right)(a, b)=0
$$

for all $x<a<b<y$ by (12), (13) and (20). Thus, (40) is equal to

$$
\begin{aligned}
& \alpha(x, x) \sum_{x<b \leq y} \beta(b, y)\left(L\left(e_{x}\right)(x, b)+L\left(e_{b y}\right)(x, y)\right) \\
& \quad+\beta(y, y) \sum_{x \leq a<y} \alpha(x, a)\left(L\left(e_{x a}\right)(x, y)+L\left(e_{y}\right)(a, y)\right) \\
& =\alpha(x, x) \beta(y, y)\left(L\left(e_{x}\right)(x, y)+L\left(e_{y}\right)(x, y)\right) \\
& \quad+\alpha(x, x) \sum_{x<b<y} \beta(b, y)\left(L\left(e_{x}\right)(x, b)+L\left(e_{b y}\right)(x, y)\right) \\
& \quad+\beta(y, y) \alpha(x, x)\left(L\left(e_{x}\right)(x, y)+L\left(e_{y}\right)(x, y)\right) \\
& \quad+\beta(y, y) \sum_{x<a<y} \alpha(x, a)\left(L\left(e_{x a}\right)(x, y)+L\left(e_{y}\right)(a, y)\right)
\end{aligned}
$$

In view of (20), we know that (41) and (43) are zero. Since

$$
\begin{aligned}
& L\left(e_{x}\right)(x, b)+L\left(e_{b y}\right)(x, y)=L\left(e_{x}\right)(x, b)+L\left(e_{b}\right)(x, b)=0, \\
& L\left(e_{x a}\right)(x, y)+L\left(e_{y}\right)(a, y)=L\left(e_{a}\right)(a, y)+L\left(e_{y}\right)(a, y)=0
\end{aligned}
$$


thanks to (12), (13) and (20), (42) and (44) are zero as well. It follows that (40) is zero.

Combining the result of the previous paragraph with (37)-(39), we conclude that

$$
\begin{aligned}
(d(\alpha) \beta+\alpha d(\beta))(x, y)= & (\alpha \beta)(x, y) L\left(e_{x y}\right)(x, y)+\sum_{x<u \leq y}(\alpha \beta)(u, y) L\left(e_{u y}\right)(x, y) \\
& +\sum_{x \leq v<y}(\alpha \beta)(x, v) L\left(e_{x v}\right)(x, y) \\
= & d\left(\left.(\alpha \beta)\right|_{x} ^{y}\right)(x, y),
\end{aligned}
$$

the latter being $d(\alpha \beta)(x, y)$ in view of Lemma 2.4 applied to $d$, whence (27).

Theorem 2.13. Let $L$ be a Lie $n$-derivation of $F I(P, R)$. If $R$ is $(n-1)$-torsion free, then $L=d+\tau$, where $d$ is a derivation of $F I(P, R)$ and $\tau$ is a central-valued linear mapping which annihilates all the $n$-th commutators.

Proof. See Definition 2.10 and Lemmas 2.11 and 2.12.

The following example shows that the assumption of $(n-1)$-torsion free in Theorem 2.13 is necessary.

Example 2.14. Let $n \geq 3, P=\{1,2\}$ with $1<2$ and char $R=n-1$. Then there are non-proper Lie $n$-derivations of $F I(P, R)$.

Proof. Define $L$ to be the identity mapping $F I(P, R) \rightarrow F I(P, R)$. Then

$$
L\left(p_{n}\left(\alpha_{1}, \ldots, \alpha_{n}\right)\right)=\sum_{i=1}^{n} p_{n}\left(\alpha_{1}, \ldots, \alpha_{i-1}, L\left(\alpha_{i}\right), \alpha_{i+1}, \ldots, \alpha_{n}\right)
$$

is equivalent to $(n-1) p_{n}\left(\alpha_{1}, \ldots, \alpha_{n}\right)=0$, which is true under the assumption char $R=n-1$.

Suppose that $L=d+\tau$ for some derivation $d$ and central-valued linear mapping $\tau$. Then

$$
e_{1}=L\left(e_{1}\right)=d\left(e_{1}\right)+\tau\left(e_{1}\right) .
$$

Since $\tau\left(e_{1}\right) \in Z(F I(P, R))$, we have $\tau\left(e_{1}\right)(1,1)=\tau\left(e_{1}\right)(2,2)$, whence

$$
d\left(e_{1}\right)(1,1)=1-\tau\left(e_{1}\right)(1,1)=1-\tau\left(e_{1}\right)(2,2) \neq-\tau\left(e_{1}\right)(2,2)=d\left(e_{1}\right)(2,2) .
$$

However,

$$
d\left(e_{1}\right)=d\left(e_{1}^{2}\right)=e_{1} d\left(e_{1}\right)+d\left(e_{1}\right) e_{1},
$$

so $d\left(e_{1}\right)(2,2)=0$ and $d\left(e_{1}\right)(1,1)=2 d\left(e_{1}\right)(1,1)$, which again implies that $d\left(e_{1}\right)(1,1)=$ 0 , a contradiction.

Problem 2.15. We would like to point out that all involved Lie-type derivations in our current work are linear. It is natural to ask whether Theorem 2.13 holds without the assumption of additivity. That is, we can investigate multiplicative Lie-type derivations of finitary incidence algebras, which is motivated by [7, 14, 37].

\section{ACKNOWLEDGEMENTS}

Mykola Khrypchenko was partially supported by the Foreign High-level Cultural and Educational Experts Project of the Beijing Institute of Technology. 


\section{REFERENCES}

[1] I. Z. Abdullaev, n-Lie derivations on von Neumann algebras, Uzbek. Mat. Zh., 5-6 (1992), 3-9.

[2] K. Baclawski, Automorphisms and derivations of incidence algebras, Proc. Amer. Math. Soc., 36 (1972), 351-356.

[3] D. Benkovič, Lie derivations on triangular matrices, Linear Multilinear Algebra, 55 (2007), 619-626.

[4] D. Benkovič, Lie triple derivations of unital algebras with idempotents, Linear Multilinear Algebra, 63 (2015), 141-165.

[5] D. Benkovič, Lie triple derivations on triangular matrices, Algebra Colloq., 18 (2011), Special Issue no. 1, 819-826.

[6] D. Benkovič and D. Eremita, Commuting traces and commutativity preserving maps on triangular algebras, J. Algebra, 280 (2004), 797-824.

[7] D. Benkovič and D. Eremita, Multiplicative Lie n-derivations of triangular rings, Linear Algebra Appl., 436 (2012), 4223-4240.

[8] M. Brešar, Commuting traces of biadditive maps, commutativity-preserving maps and Lie maps, Trans. Amer. Math Soc. 335 (1993), 525-546.

[9] M. Brešar, Commuting maps: a survey, Taiwanese J. Math. 8 (2004), 361-397.

[10] R. Brusamarello, E. Fornaroli and E. Santulo Jr, Classification of involutions on finitary incidence algebras, Int. J. Algebra Comp. 24 (2014), 1085-1098.

[11] R. Brusamarello and D. Lewis, Antomorphisms and involutions on incidence algebras, Linear Multilinear Algebra, 59 (2011), 1247-1267.

[12] W.-S. Cheung, Lie derivations of triangular algebras, Linear Multilinear Algebra, 51 (2003), 299-310.

[13] Y.-Q. Du and Y. Wang, Lie derivations of generalized matrix algebras, Linear Algebra Appl., 437 (2012), 2719-2726.

[14] A. Fošner, F. Wei and Z.-K. Xiao, Nonlinear Lie-type derivations of von Neumann algebras and related topics, Colloq. Math., 132 (2013), 53-71.

[15] I. N. Herstein, Lie and Jordan structures in simple associative rings, Bull. Amer. Math. Soc. 67 (1961), 517-531.

[16] N. S. Khripchenko, Automorphisms of finitary incidence rings, Algebra Discrete Math., 9 (2010), 78-97.

[17] N. S. Khripchenko, Derivations of finitary incidence rings, Comm. Algebra, 40 (2012), 2503-2522.

[18] N. S. Khripchenko and B. V. Novikov, Finitary incidence algebras, Comm. Algebra, 37 (2009), 1670-1676.

[19] M. Khrypchenko, Jordan derivations of finitary incidence rings, Linear Multilinear Algebra, 64 (2016), 2104-2118.

[20] M. Khrypchenko, Local derivations of finitary incidence rings, Acta Math. Hungar., 154 (2018), 48-55.

[21] M. Koppinen, Automorphisms and higher derivations of incidence algebras, J. Algebra, 174 (1995), 698-723.

[22] F.-Y. Lu, Lie derivations of certain CSL algebras, Israel J. Math., 155 (2006), 149-156.

[23] F.-Y. Lu, Lie derivations of $\mathcal{J}$-subspace lattice algebras, Proc. Amer. Math. Soc., 135 (2007), 2581-2590.

[24] F.-Y. Lu and B.-H. Liu, Lie derivations of reflexive algebras, Integr. Equ. Oper. Theory, 64 (2009), 261-271. 
[25] W. S. Martindale III, Lie derivations of primitive rings, Michigan Math. J. 11 (1964), 183-187.

[26] M. Mathieu and A. R. Villena, The structure of Lie derivations on $C^{*}$-algebras, J. Funct. Anal. 202 (2003), 504-525.

[27] C. R. Miers Lie derivations of von Neumann algebras, Duke Math. J., 40 (1973), 403-409.

[28] C. R. Miers Lie triple derivations of von Neumann algebras, Proc. Amer. Math. Soc., 71 (1978), 57-61.

[29] X.-F. Qi, Characterizing Lie n-derivations for reflexive algebras, Linear Multilinear Algebra, 63 (2015), 1693-1706.

[30] X.-F. Qi, Lie n-derivations on $\mathcal{J}$-subspace lattice algebras, Proc. Indian Acad. Sci. Math. Sci., 127 (2017), 537-545.

[31] E. Spiegel and C. O'Donnell, Incidence algebras, Monographs and Textbooks in Pure and Applied Mathematics, vol. 206, Marcel Dekker, New York, 1997.

[32] E. Spiegel, On the automorphisms of incidence algebras, J. Algebra, 239 (2001), 615-623.

[33] R. Stanley, Structure of incidence algebras and their automorphism groups, Bull. Amer. Math. Soc. 76 (1970), 1236-1239.

[34] R. Stanley, Enumerative Combinatorics, vol. 1. With a foreword by GianCarlo Rota. Cambridge Studies in Advanced Mathematics, vol. 49, Cambridge University Press, Cambridge, 1997.

[39] D.-N. Wang and Z.-K. Xiao, Lie triple derivations of incidence algebras, To appear in Comm. Algebra, http://arXiv:1901.05690v1 [math.RA]

[36] Y. Wang, Lie n-derivations of unital algebras with idempotents, Linear Algebra Appl., 458 (2014), 512-525.

[37] Y. Wang and Y. Wang, Multiplicative Lie n-derivations of generalized matrix algebras, Linear Algebra Appl., 438 (2013), 2599-2616.

[38] M. Ward, Arithmetic functions on rings, Ann. Math. 38 (1937), 725-732.

[39] Z.-K. Xiao, Jordan derivations of incidence algebras, Rocky Mountain J. Math. 45 (2015), 1357-1368.

[40] Z.-K. Xiao and F. Wei, Lie triple derivations of triangular algebras, Linear Algebra Appl., 437 (2012), 1234-1249.

[41] X. Zhang and M. Khrypchenko, Lie derivations of incidence algebras, Linear Algebra Appl., 513 (2017), 69-83.

Departamento de Matemática, Universidade Federal de Santa Catarina, Campus Reitor João David Ferreira lima, Florianópolis, SC, CEP: 88040-900, BraziL

E-mail address: nskhripchenko@gmail.com

School of Mathematics and Statistics, Beijing Institute of Technology, Beijing 100081, P. R. CHINA

E-mail address: daoshuo@hotmail.com, daoshuowei@gmail.com 